\title{
Programme frequency, type, time and duration do not explain the effects of balance exercise in older adults: a systematic review with a meta-regression analysis
}

\author{
Melanie K Farlie, ${ }^{\oplus 1,2}$ Lauren Robins, ${ }^{1,2}$ Romi Haas, ${ }^{1,2}$ Jennifer L Keating, \\ Elizabeth Molloy, ${ }^{3}$ Terry P Haines ${ }^{1,2}$
}

\begin{abstract}
- Additional material is published online only. To view please visit the journal online (http://dx.doi.org/10.1136/ bjsports-2016-096874).
\end{abstract}

${ }^{1}$ Faculty of Medicine, Nursing and Health Sciences, Monash University, Frankston, Victoria, Australia

${ }^{2}$ Allied Health Research Unit, Monash Health, Cheltenham, Victoria, Australia ${ }^{3}$ Department of Medical Education, The University of Melbourne, Melbourne, Victoria, Australia

Correspondence to Melanie K Farlie, Faculty of Medicine, Nursing and Health Sciences, Monash University, Frankston, Victoria 3199 Australia;

melanie.farlie@monash.edu

Accepted 2 January 2018 Published Online First 25 January 2018
Check for updates

To cite: Farlie MK, Robins L, Haas R, et al. Br J Sports Med 2019:53:996-1002.

\section{ABSTRACT}

Objective The objective of this systematic review was to examine the effects of different balance exercise interventions compared with non-balance exercise controls on balance task performance in older adults. Design Systematic review.

Data sources Medline, Cumulative Index to Nursing and Allied Health Literature, EMBASE, Scopus and Cochrane Database of Systematic Reviews were searched until July 2017.

Eligibility criteria for selecting studies Systematic reviews and meta-analyses of randomised trials of balance exercise interventions for older adults were identified for extraction of eligible randomised trials. Eligibility criteria for inclusion of randomised trials in meta-analyses were comparison of a balance exercise intervention with a control group that did not perform balance exercises, report of at least one end-intervention balance outcome measurement that was consistent with the five subgroups of balance exercise identified, and full-text article available in English.

Results Ninety-five trials were included in metaanalyses and 80 in meta-regressions. For four balance exercise types (control centre of mass, multidimensional, mobility and reaching), significant effects for balance exercise interventions were found in meta-analyses (standardised mean difference (SMD) 0.31-0.50), however with considerable heterogeneity in observed effects ( $\left.I^{2}: 50.4 \%-80.6 \%\right)$. Risk of bias assessments (Physiotherapy Evidence Database score and funnel plots) did not explain heterogeneity. One significant relationship identified in the meta-regressions of SMD and balance exercise frequency, time and duration explained $2.1 \%$ of variance for the control centre of mass subgroup.

Conclusion Limitations to this study included the variability in design of balance interventions, incomplete reporting of data and statistical heterogeneity. The design of balance exercise programmes provides inadequate explanation of the observed benefits of these interventions.

\section{INTRODUCTION}

Fall-related injury and death are a leading cause of morbidity and mortality in older adults around the world. ${ }^{1}$ There have been many studies of exercise interventions to improve balance in older people. ${ }^{2}$ Balance exercises improve balance and reduce falls ${ }^{23}$; however, the reporting of exercise prescription variables has been incomplete. ${ }^{4}$ Balance exercise to date has been poorly defined and described using variable terminology, such as balance enhancing exercise, ${ }^{5}$ balance training, ${ }^{6}$ proprioceptive training ${ }^{7}$ and neuromuscular training. ${ }^{8}$ Common to these terms is the definition of exercises that challenge the postural control system. For the purposes of this review, we use the term balance exercise to encompass all forms of training of this type.

The four variables routinely manipulated in exercise prescription are frequency, intensity, type and time spent exercising. Manipulation of these variables to achieve exercise effects is known as the FITT (frequency, intensity, type, time) principle. ${ }^{9}$ Balance exercise programme frequency (eg, sessions/week), type (eg, static balance-controlling the centre of mass (COM) over a static base of support; dynamic balance-stepping/mobility challenges; multidimensional-ie, tai chi) and time (eg, within session time measured in minutes and programme duration usually reported in weeks) are readily measurable and reported. A recent systematic review of randomised controlled trials of balance exercise interventions identified an absence in the reporting of balance exercise intensity. In addition, no instrument to rate the intensity of balance challenge intensity was identified. ${ }^{4}$ This finding exposes a limitation in the study of balance exercise programme efficacy. It is unknown whether the frequency, type, time and duration of balance exercise programmes alone can explain the effect of balance exercise programmes on balance performance and falls incidence.

Without a valid and reliable measure of balance exercise intensity, the dose-response relationship of balance exercise, and the role of intensity, is still to be fully investigated. Studies of dose-response in aerobic and resistance exercise training have found effective minimum thresholds for these exercise types. For aerobic exercise this is $150-300 \mathrm{~min} /$ week at moderate intensity or $75-150 \mathrm{~min} /$ week at vigorous intensity. ${ }^{5}$ For strength training this is 2-3 sessions per week at high intensity. ${ }^{5}$ For balance exercise dosage, recommendations rely on prescription of exercise frequency, type and time. In the absence of a measure of intensity, balance exercise is generally prescribed 2-3 times per week for 45-60 min, with the most difficult tasks practised 2-3 times per session; however, this is based on expert opinion rather than research evidence. ${ }^{510} 11$

It may be that examination of the variables of frequency, type, time and duration is sufficient for determining the minimum effective thresholds of 
balance exercise to improve performance on balance outcome measures. If these factors are sufficient, then meta-regression of these factors should satisfactorily explain the variance in outcomes from trials of interventions to improve balance.

\section{Objective of the review}

The aim of this systematic review was to examine the effects of different balance exercise interventions compared with non-balance exercise controls on balance task performance in older adults. Extracted data were grouped by balance exercise type with matched balance outcome measures. The magnitude of overall programme effects and effects relative to the variables of frequency, type, time and duration was analysed.

\section{METHODS}

Randomised controlled trial identification and selection

Balance exercise intervention trials in older adults have been reported in randomised trials of exercise interventions alone, and as part of a broader 'package' of interventions aimed at improving balance and preventing falls. For this reason, a search for randomised trials of balance exercise interventions is potentially not broad enough to capture the trials that test the efficacy of balance exercise as a secondary intervention in this population. Therefore, a two-stage strategy of identifying systematic reviews and meta-analyses that investigated balance outcomes and exercise ( \pm other) interventions was conducted first, with subsequent identification of eligible randomised trials from relevant review articles. Review articles were included for examination if they included at least one randomised trial examining the effect of a balance exercise intervention in an older adult population. The balance exercise intervention could be either a primary or secondary intervention. Randomised trials cited in earlier reviews were included in this review if they investigated a balance exercise intervention compared with a non-balance exercise control, reported at least one balance outcome at baseline and at the end-intervention time point, and included a majority of older adults aged $\geq 55$ years. A previous review using this systematic search procedure was assessed using capture-recapture analysis and estimated a trial identification depth of $92 \%$ (148 of a possible 162 trials). ${ }^{4}$

Searches to identify randomised trials for inclusion in this review were completed on 20 July 2017 by the lead investigator (MKF). The searches for systematic reviews were restricted to 2006 onwards as it was expected that any randomised trials from reviews prior to 2006 were highly likely to be included in reviews in the subsequent 12-year period. Searches were performed on Medline (1946 to present and in-process and other non-indexed citations), Cumulative Index to Nursing and Allied Health Literature, EMBASE, Scopus and Cochrane Database of Systematic Reviews. Search terms included combinations of older adult/ person, elderly, geriatric, aged, balance, exercise, train, physical

Table 1 OVID Medline (1946 to present and in-process and other non-indexed citations) search strategy with truncation

\begin{tabular}{|c|c|c|c|c|c|c|}
\hline OR & & & & OR & & Limitations \\
\hline $\begin{array}{l}\text { older adult\$ } \\
\text { older person } \\
\text { elderly } \\
\text { geriatric } \\
\text { aged }\end{array}$ & AND & Balance \$ & AND & $\begin{array}{l}\text { exercis\$ } \\
\text { train\$ } \\
\text { physical } \\
\text { active\$ } \\
\text { physical } \\
\text { intervention }\end{array}$ & AND & $\begin{array}{l}\text { ab, ti } \\
\text { Meta analysis } \\
\text { OR } \\
\text { 'review' } \\
\text { '2006- current' } \\
\text { 'review } \\
\text { articles' }\end{array}$ \\
\hline
\end{tabular}

Box Eligibility criteria for step 1 (search for systematic reviews and meta-analyses) and step 2 (extraction of randomised controlled trials reporting balance exercise interventions) used in this review

Step 1: systematic reviews and meta-analyses

- Systematic reviews and meta-analyses that included randomised controlled trials of balance exercise training interventions or mixed interventions that included balance exercise training.

- Publication date from January 2006.

- Majority of trial participants are older adults ( $>55$ years).

Step 2: randomised controlled trials

- Randomised controlled trial.

- Balance exercise intervention group compared with a control group that did not perform balance exercises.

- Outcomes included a balance outcome measurement.

- Follow-up measure at end-intervention time point reported for baseline balance outcomes (mean and SD, or data from which means or SD could be calculated, eg, SE and CIs).

- Full text available.

Article published in English.

intervention and activity. Exact search terms with truncation used in Medline are detailed in table 1 as example.

The title and abstract of review articles were examined by the lead investigator (MKF) against the eligibility criteria reported at step 1 in the box. Reference lists of eligible reviews were also examined to identify any additional reviews that met the eligibility criteria based on the title and the abstract. Citations for all randomised trials reported in eligible review articles were extracted, and a review of title and the abstract was performed independently by investigators MKF and LR and/or RH to evaluate if trials met the eligibility criteria reported at step 2 in the box. Reference lists of eligible trials were also reviewed to identify any additional trials meeting the eligibility criteria for inclusion in this review based on the title and the abstract. Full-text review was then performed independently by investigators MKF and LR and/or RH to confirm eligibility of each trial prior to data extraction. At each stage of trial identification and selection, discrepancies were resolved by discussion to the point of consensus in all instances.

\section{Data extraction}

Data were extracted independently by investigators MKF and LR and/or RH. The results of data extraction were then compared and merged by the three investigators to create the master data set. All discrepancies were resolved by discussion and consensus. The categorisation of extracted data was based on the principle of training specificity. Balance training may include various types of activities that require coordination of multiple body systems (eg, proprioception, vestibular, vision, neuromuscular), and training needs to be specific to the type of balancing task that is being targeted (eg, maintaining balance over fixed base of support). ${ }^{12}$ Options for the measurement of balance performance also vary from global batteries of tests, such as the Berg Balance Scale, ${ }^{13}$ to measurements of domains of balance performance, such as reaching, mobility tasks or controlling the COM over the base of support. Applying the principle of specificity to balance training recognises that practising different types of balance tasks is likely to show most, 
Table 2 Classifications of types of balance exercise interventions and balance outcome measures

\begin{tabular}{|c|c|c|}
\hline Classification & Interventions & Outcome measures \\
\hline Multidimensional & $\begin{array}{l}\text { Functional exercise programmes, } \\
\text { tai chi, ball/balloon games }\end{array}$ & $\begin{array}{l}\text { For example, Berg Balance } \\
\text { Scale }\end{array}$ \\
\hline Reaching & Reaching exercises & For example, functional reach \\
\hline $\begin{array}{l}\text { Control centre of } \\
\text { mass }\end{array}$ & $\begin{array}{l}\text { Static balance exercises, for } \\
\text { example, single leg stance, } \\
\text { balance platform interventions } \\
\text { where base is kept static }\end{array}$ & $\begin{array}{l}\text { For example, Clinical Test of } \\
\text { the Sensory Integration of } \\
\text { Balance }\end{array}$ \\
\hline Mobility & $\begin{array}{l}\text { Mobility component, such as, } \\
\text { obstacle courses }\end{array}$ & $\begin{array}{l}\text { For example, Timed-Up and } \\
\text { Go Test }\end{array}$ \\
\hline Stepping & Stepping exercises & For example, Step test \\
\hline
\end{tabular}

if not all, improvement on balance outcome measures that are matched to the type of balance task trained. In other words, you would not expect a balance exercise programme that involved only the performance of multidirectional stepping practice to result in an improvement in an outcome measure that assessed reaching ability, such as the Functional Reach Test. ${ }^{14}$ Equally a multidimensional balance exercise programme such as tai chi could conceivably result in improvement in multiple domains of balance performance, so improvement would be most appropriately assessed using a global multidimensional outcome measure, such as the Berg Balance Scale. To guide the categorisation of balance tasks and measurements, subcategories described in the classifications for the Balance Outcome Measure for Elder Rehabilitation ${ }^{15}$ were considered. These categories, along with the addition of a 'multidimensional' classification to encompass interventions such as tai chi, dance and ball or balloon games, were used to categorise the balance exercise interventions and measurements extracted from trials in this review. Classifications of interventions and matching outcomes are summarised in table 2.

For each type of balance exercise (multidimensional, reaching, control COM, mobility and stepping), the programme frequency, time and duration were extracted. For 'mixed programs', those not solely balance training (ie, programmes that included strength, endurance and/or flexibility, as well as balance exercises), these variables were separated. For example, if the exercise session was $60 \mathrm{~min}$ long, but only $10 \mathrm{~min}$ were spent on balance exercise, the balance exercise session time was recorded as $10 \mathrm{~min}$. If balance exercises only occurred once a week in a $3 \times /$ week programme, the frequency was recorded as $1 \times /$ week and so on. If more than two groups were described, data were extracted for the balance intervention group and the inactive control group.

When more than one classification of balance outcome measure was reported for the same participants, data for each outcome measure classification were extracted separately for subgroup analysis. In anticipation that multiple outcome measures matched to the same type of balance exercise could be reported (eg, the effect of a control COM intervention being measured using single leg stance and anteroposterior sway), we determined a preferred order of outcome extraction a priori to avoid data extraction for multiple outcomes from the same study being entered into the subgroup meta-analysis, and to extract the same outcome measure from studies for pooling in meta-analyses wherever possible (online supplementary material 1).

Means and SD were extracted at baseline and the end-intervention time points. Differences between groups were expressed as standardised mean differences (SMD). Where possible, SDs were calculated from means and SEs or CIs. To minimise the risk of producing spurious findings and uncertainty associated with imputed results, studies that reported insufficient outcome data to allow calculation of means and SDs (eg, medians and IQRs, change scores or stratified outcomes) were not included in analyses. ${ }^{16}$ All authors of studies that reported adequate outcome data for inclusion in meta-analysis but had not fully reported balance exercise programme variables required for meta-regression analysis (most commonly the number of minutes dedicated to balance exercise training within a session) were contacted for additional information, and when supplied these data were also included in the final data set.

\section{Risk of bias assessments}

Study-level risk of bias was assessed using the Physiotherapy Evidence Database (PEDro) scale, an 11-item scale scored out of 10 that assesses potential risks for bias in the reporting of clinical trials. A PEDro score of $6 / 10$ or higher is considered to indicate good to excellent methodological quality. ${ }^{17}$ Each trial was rated independently by MKF and LR and/or RH. Any discrepancies in PEDro ratings were resolved through discussion to the point of consensus. After reaching consensus, in addition to investigator scoring, each study was cross-checked with ratings recorded in the PEDro database. Potential for bias was further assessed following meta-analysis by generating funnel plots for all analyses with greater than 10 studies, as recommended by Higgins and Green. ${ }^{16}$

\section{Data analysis}

Study-level risk of bias was examined using the regression plots of PEDro scores and the magnitude of observed effects (SMD) to determine if there was any correlation between PEDro score and trial outcome. A significant correlation may indicate methodological diversity contributing to bias at the study level (eg, lack of intention-to-treat analysis or non-blinding of outcome assessors) and influencing trial outcomes.

SMDs between groups and the SE in the estimate of the SMD (seSMD) were calculated for generation of meta-analyses using the five balance exercise types as the independent variable (multidimensional, control COM, mobility, reaching and stepping) and the corresponding balance outcomes data as the dependent variable. Outcomes were pooled using random-effects meta-analysis given the non-uniform outcome measures used between trials. Statistical heterogeneity, indicative of between-study variability in observed effects beyond chance, was examined using the $\mathrm{I}^{2}$ statistic. Funnel plots were generated, by plotting SMD against the inverse of seSMD for each meta-analysis of more than 10 studies, to visually examine for asymmetry and assess the potential for possible publication bias affecting the meta-analysis outcome. $^{18}$

Single variable regression bubble plots were generated initially to examine the relationships between individual variables and their effect on outcome measurements (SMD) that were subsequently examined in the multiple variable meta-regression analyses. Examination of single variable plots prior to meta-regression is needed to determine if any variables have a significant association with study effects (SMD) when examined in isolation. If a single variable has a significant association with study effect prior to inclusion in a meta-regression analysis, inclusion of this variable could increase the risk of misleading findings. ${ }^{19}$ Meta-regression analyses were constructed using the theoretical models of the FITT 
SYSTEMATIC REVIEWS (SRs) AND METAANALYSES (M-As)
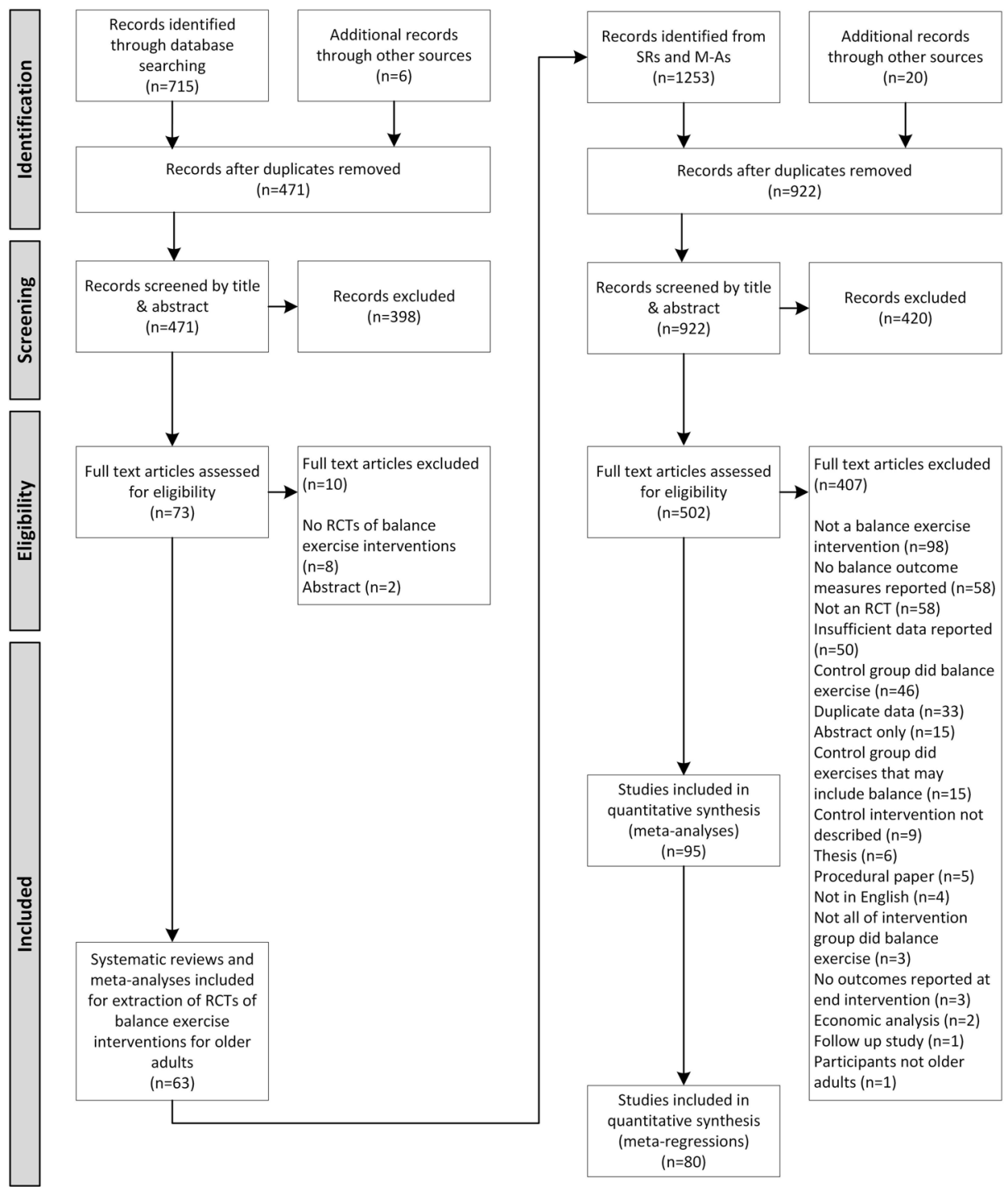

Figure 1 Flow of studies through the review. Initial search for systematic reviews and meta-analyses that included randomised controlled trials (RCTs) of balance exercise interventions, followed by extraction of eligible RCTs from eligible review articles.

principle and exercise specificity. Given that balance exercise intensity cannot currently be quantified, having no validated measure, ${ }^{4}$ the meta-regression model was built using the available balance exercise programme variables that were reported: frequency (sessions/week), balance exercise type (eg, control COM, reaching, mobility), within-session balance exercising time (min) and programme duration (weeks) as independent variables in meta-regression analyses. We used random-effects meta-regression, employing restricted maximum likelihood, in calculating the SEs of the effect estimates. Meta-regression analysis was conducted on balance exercise programme types where greater than 20 observations were available, as recommended by Gagnier et al. ${ }^{20}$ This was to reduce the risk of generating spurious significant findings from overfit of the meta-regression analysis based on the ratio of the number of studies to the number of covariates in the model.

For each meta-regression the regression coefficient, 95\% upper and lower CIs and P values were reported, along with $\mathrm{I}^{2}$ and adjusted $\mathrm{R}^{2}$ values. The adjusted $\mathrm{R}^{2}$ values were used to evaluate to what degree variables included in the model explained the variance in trial outcomes. Funnel plots were generated using RevMan ${ }^{21}$; all other statistical analyses were conducted using Stata V.14.

\section{RESULTS}

\section{Study characteristics}

The flow of the studies through the review is shown in figure 1. Examination of 63 systematic reviews (online supplementary material 2) led to the identification of 95 randomised trials for inclusion in meta-analyses (online supplementary material 3). The number of participants in included trials ranged from 727 for stepping interventions, to 4479 for control COM interventions. Intervention group sample size ranged from 9 to 313 participants. Average age across the five exercise types was 74.5 years (range 59-88). One hundred and sixty outcome measurements were reported across the five balance exercise types (eg, a balance exercise programme with reaching exercises reported a reaching outcome measure). 
Exercise variables, balance outcome measures and risk of bias data extracted from the 95 randomised trials were tabulated for analysis (online supplementary materials 4 and 5).

\section{Evaluation of potential sources of bias in included trials}

The study PEDro total scores for included trials ranged from 3 to 9 , with $54 \%$ of trials controlling for six or more potential sources of bias. Given that these were exercise intervention trials, the subject blinding and therapist blinding criteria were not met for any included trials. The next three least met criteria were concealed allocation (30.5\%), intention-to-treat analysis (38.9\%) and assessor blinding (56.8\%). Regression plots generated for each exercise type showed no significant correlation between PEDro scores and observed effects (SMD) for any intervention type (multidimensional $\mathrm{r}=-0.24, \mathrm{P} 0.16$; reaching $\mathrm{r}=0.23, \mathrm{P} 0.34$; control $\mathrm{COM} \mathrm{r}=-0.20, \mathrm{P} 0.12$; mobility $\mathrm{r}=-0.10, \mathrm{P} 0.50$; stepping $\mathrm{r}=-0.17$, P 0.74 ) (online supplementary material 5). This indicated that risk of outcomes showing systematic bias related to the quality of trial methodology was low, and therefore all identified trials were included in meta-analyses. Funnel plots indicated a reasonable spread of effect sizes around the mean effect, so any influence that publication bias might have appears to be relatively small ${ }^{18}$ (online supplementary material 6).

\section{Effectiveness of balance exercise by type}

Meta-analyses of balance exercise interventions showed small to moderate effects in favour of exercise programmes that included multidimensional (SMD 0.50, CI 0.36 to 0.65 ), reaching (SMD 0.48 , CI 0.33 to 0.64 ), control COM (SMD 0.42, CI 0.27 to 0.56 ) and mobility (SMD 0.31 , CI 0.20 to 0.43 ) balance training. Meta-analysis of stepping interventions favoured the non-balance exercise control (SMD $0.25,-0.08$ to 0.57 ). Substantial between-study heterogeneity was evident for all interventions, with the $\mathrm{I}^{2}$ statistic ranging from moderate and significant (reaching 50.4\%, $\mathrm{P}=0.006$; multidimensional 62.3\%, $\mathrm{P}<0.001$; mobility $62.7 \%, \mathrm{P}<0.001$; and stepping $67.3 \%, \mathrm{P}=0.009)$ to high and significant (control COM 80.6\%, P<0.001), indicating the level of variability in effect estimates attributable to study characteristics beyond that expected by chance ${ }^{18}$ (online supplementary material 6).

\section{Examination of trial outcome variance by prescription variable}

Further inspection of extracted data using the same exercise type and outcome measure pairings established that all balance exercise prescription variables (frequency, type, time, duration) were reported for 80 of the 95 studies that were included in the meta-analyses. One hundred and thirty-three outcomes from the 80 studies were available for inclusion in meta-regression analyses (figure 1). Complete data for 20 or more observations were available for meta-regression of only three intervention subgroups: multidimensional $(n=27)$, control COM $(n=51)$ and mobility $(n=35)$. Reaching and stepping intervention data were only available for 15 and 5 studies, respectively, and thus excluded from meta-regression analysis.

Single variable regression plots were generated prior to meta-regression to explore the relationship between the balance exercise variables frequency, exercise time and programme duration by corresponding outcome measures (SMDs) for the three exercise types (online supplementary material 7). No significant association was found between individual exercise prescription variables and study effects (SMD) for any type of exercise.
Subsequent meta-regression analysis examining the relationship between exercise intervention type, session frequency, time, duration and study effects (SMD) was executed. Meta-regression analyses shown in table 3 found moderate to high statistical heterogeneity $\left(\mathrm{I}^{2}\right.$ range $\left.65.1 \%-81.9 \%\right)$, which as expected was similar to the results obtained in the meta-analyses. There were no significant relationships identified between SMD and frequency, type, time or duration for the mobility or multidimensional exercise programmes. A significant relationship was found between SMD and exercise type for the control COM exercise programmes ( $\mathrm{P}$ 0.049), indicating that control $\mathrm{COM}$ exercises are associated with improved performance on control COM outcome measurements regardless of programme frequency, time or duration. However, the adjusted $\mathrm{R}^{2}$ for the control COM analysis was $2.15 \%$, so this significant result fails to account for $97.85 \%$ of the variance in the model. Adjusted $\mathrm{R}^{2}$ was $-7.71 \%$ and $-1.22 \%$, respectively, for the multidimensional and mobility programme analyses, indicating that no variables explain the variation in intervention effect between studies for these exercise types. Negative adjusted $\mathrm{R}^{2}$ values indicate that the factors included in the model actually 'explain less of the heterogeneity than would be expected by chance' (p. 500). ${ }^{22}$

\section{DISCUSSION}

This review has shown that when most types of balance exercise interventions are pooled for analysis, there is a small $(\mathrm{SMD}<0.4)$ to moderate (SMD $0.4-0.7)^{16}$ positive effect on balance performance in older adults. This finding needs to be interpreted with caution due to the moderate to high degree of unexplained heterogeneity found in all analyses $\left(\mathrm{I}^{2}\right.$ : $\left.50.4 \%-80.6 \%\right)$. The results of this review are consistent with those of a smaller review of 23 trials recently published that used similar groupings of balance exercise types and outcome measures. ${ }^{6}$ That review was limited to balance exercise-only interventions compared with non-exercise controls in healthy community-dwelling older adults and reported pooled effect sizes ranging from SMD 0.51 to 1.73 and $\mathrm{I}^{2}$ values ranging from $76 \%$ to $92 \%$. $^{6}$

Meta-regression analyses conducted as part of this review have demonstrated no association between mobility and multidimensional exercise programme frequency, type, time or duration (examined alone or in combination) with balance outcomes and an association between control COM exercise type and outcomes that explains just $2.15 \%$ of the variance observed. These analyses are based on 27-51 studies, which is greater than the minimum number of 20 observations recommended for meta-regression analysis. ${ }^{20}$ Given this theorised minimum is somewhat arbitrary, this result warrants cautious interpretation, but it appears to indicate a lack of support for the frequency, type and time components of the FITT exercise prescription principle when applied to balance exercise programmes. There are two competing explanations for this. First, that the individual frequency, type and time components of the FITT exercise prescription principle are not uniquely important in prescribing balance exercises to older adults. This would seem at odds with other meta-analyses that have identified the importance of these factors, where exercise programmes for the prevention of falls have been considered. ${ }^{3}$ The second is that missing elements (such as intensity) of the exercises performed, which have not been reported to date, are critical factors.

This is the first known attempt to use meta-regression to analyse the relative contribution of reported exercise prescription variables (balance exercise frequency, type, time and duration) on the performance of balance outcome measurements following 


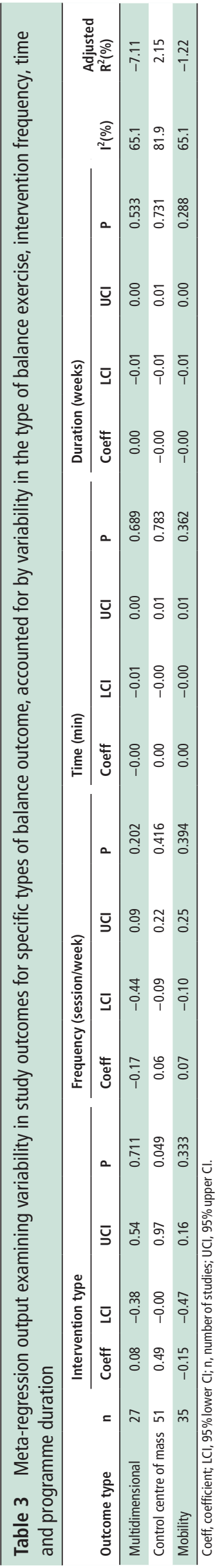

balance exercise interventions. Within a single empirical study, it is possible to study the effect of one exercise variable, for example, frequency, but only if all other variables are held constant. There is a need to specifically design studies that investigate the relative impact of different exercise prescription elements individually if we are going to enhance our understanding in this area. Analyses in the present review have sought to make use of variation in these elements across the body of literature but have been limited by the lack of reporting of the intensity variable. With the development of a measure of balance exercise intensity, future analyses could be performed incorporating intensity in the model. This would assist researchers to determine if balance exercise intensity is an important factor in balance exercise prescription. There is an urgent need to develop such a measure of balance exercise intensity and incorporate this in future studies examining balance exercise interventions in older adults.

There may be additional benefit to improved understanding and measurement of balance exercise intensity. It has been noted, specifically in relation to strength and aerobic training, that the variable of exercise intensity is 'the most closely related to serious adverse events' (p820). ${ }^{9}$ This caution could extend to balance exercise prescription given the potential risk of falls and fall-related injury with highly challenging exercises. In fact, falls and balance specialists tend to avoid prescribing challenging balance exercises to older adults engaging in unsupervised exercise programmes. ${ }^{23}$

\section{Limitations}

Limitations of the present review need to be acknowledged. First, moderate to high levels of between-study heterogeneity in effect sizes were observed. It is possible that variations in study quality may have confounded the results of the studies included in this review. This however appears unlikely as there was no association between study PEDro scores and individual study SMDs. Examination of funnel plots indicates that the risk of publication bias influencing the results is low; however, this possibility cannot be excluded particularly given only articles published in English language were included in this review. Second, data from 50 trials were insufficiently reported and not imputed for inclusion in these meta-analyses. In addition, data from several studies were unable to be incorporated into the meta-regression analyses due to incomplete reporting of the frequency, type and time variables of the multidimensional, mobility and control COM balance interventions. These considerations limit confidence in these review findings and challenge the research community to develop standards for balance exercise prescription and reporting. Similar levels of missing data in reporting of exercise trials have been reported by others with recommendations for standardised reporting. ${ }^{24}$ The recent introduction of the Template for Intervention Description and Replication guidelines ${ }^{25}$ to enhance the completeness of reporting of interventions used in randomised controlled trials may help to address this issue in future years.

Third, there were only a small number of studies reporting reaching or stepping interventions. Hence, we were unable to perform meta-regression analyses for these interventions. In addition, meta-regression analyses of pooled results from the multidimensional, control COM and mobility interventions are inferior to a direct investigation of the impact of different variable combinations within a single study. A single study would address the meta-regression limitation of between-study variability and inconsistency in control conditions potentially contaminating results. Future studies to address this limitation are needed. 
Finally, a statistical analysis based on a theoretical model may not include all possible variables in the model, nor be a close representation of how different variables interact in situ. The FITT model is however the putative model used in exercise prescription, so was an appropriate model to examine for this meta-regression analysis.

\section{CONCLUSION}

Given the public health cost and individual impact of falls and fall-related injuries in older adults, clinicians would benefit from clear evidence to guide optimal balance exercise prescription. Meta-analysis in this review showed that balance exercise programmes categorised into four subtypes (multidimensional, control COM, mobility and reaching) had a small to moderate effect on balance performance in older adults. Meta-regression of exercise variables in interventions designed to improve control of COM accounted for less than $3 \%$ of the variance in outcomes reported, and none of the variance associated with mobility or multidimensional interventions. This indicates that while balance exercise is effective at improving balance task performance across most subdomains, there is no clear evidence that specific dosage in exercise frequency, time or duration is typically associated with positive effects.

\section{RECOMMENDATIONS}

- Balance exercise programmes produce small to moderate improvements in balance of older adults.

- Clinicians seeking to improve how well older adults control their COM should prescribe exercises with focus. It is not clear whether improving other facets of balance requires the same level of specificity in exercise prescription.

- Monitor the effect of balance exercise programmes using outcome measures specific to the target skill, for example, use Functional Reach to measure the effect of a programme

\section{What is already known?}

- Balance exercises are routinely prescribed to improve balance to prevent falls in older adults.

- Exercise that is adequately challenging drives skill development.

- The most effective way to prescribe balance exercises in rehabilitation has not been established.

- Optimal exercise prescription could be built around 'frequency, intensity, time and type', but we have not established how to measure balance exercise intensity.

\section{What are the findings?}

- There is incomplete reporting of balance exercise intervention variables in randomised controlled trials of balance exercise interventions in approximately $8 \%$ of analysed studies.

- With reported exercise variables of frequency, type, exercise time and programme duration, we are unable to predict the outcome of balance exercise interventions in older adults.

- The addition of intensity (the 'I' in FITT) to a meta-regression model may or may not explain the observed effects of balance exercise interventions in older adults. If a valid measure for balance exercise intensity becomes available, this analysis should be repeated. requiring the exerciser to reach outside of their base of support.

- To assist ongoing analysis of exercise effects, when reporting results of balance exercise, trials should include specific information on the types of balance exercise performed, as well as frequency, time and duration.

Contributors All authors contributed to the conception and design of the work. MKF, LR and RH contributed to acquisition of data. All authors contributed to analysis and interpretation of data, drafting and revision of the manuscript, and gave final approval of the version to be published.

Funding MKF is supported by the Monash University Maxwell King PhD Scholarship. TPH is supported by a National Health and Medical Research Council Career Development Fellowship.

Competing interests Employment or affiliation: TPH is Director of Hospital Falls Prevention Solutions. This company provides the Safe Recovery Training Program for the purposes of preventing falls in the hospital setting. Speaker forum: TPH received payment for speaking at the Australian New Zealand Falls Prevention Conference through the Australian New Zealand Falls Prevention Society. Consultancy: TPH provides statistical analysis consultancy for DorsaVi in the field of back pain research. Expert testimony: TPH has provided expert witness testimony in the area of falls in the hospital setting through MinterEllison Lawyers. Publications: TPH has authored trials included in this study but was not involved in the evaluation of those trials for the purpose of this study.

Provenance and peer review Not commissioned; externally peer reviewed. Data sharing statement All data available in the online supplementary files.

(C) Article author(s) (or their employer(s) unless otherwise stated in the text of the article) 2019. All rights reserved. No commercial use is permitted unless otherwise expressly granted.

\section{REFERENCES}

1 World Health Organization. Fact sheet N³44. Secondary fact sheet $N^{\circ} 344$ October 2012. 2012 http://www.who.int/mediacentre/factsheets/fs344/en/ (accessed 17 Aug 2013).

2 Howe TE, Rochester L, Neil F, et al. Exercise for improving balance in older people. Cochrane Database Syst Rev 2011:CD004963

3 Sherrington C, Tiedemann A, Fairhall N, et al. Exercise to prevent falls in older adults: an updated meta-analysis and best practice recommendations. N S W Public Health Bull 2011;22:78-83.

4 Farlie M, Robins L, Keating J, et al. An absence of intensity reporting in the prescription of balance exercises in randomised controlled trials: a systematic review. J Physiother 2013;59:227-35.

5 American College Sports Medicine. ACSM's resource manual of guidelines for exercise testing and prescription. 7th edn. Philadelphia: Wolters Kluwer Lippincott Williams and Wilkins, 2014.

6 Lesinski M, Hortobágyi T, Muehlbauer T, et al. Effects of balance training on balance performance in healthy older adults: a systematic review and meta-analysis. Sports Med 2015:45:1721-38

7 Silva KN, Mizusaki Imoto A, Almeida GJ, et al. Balance training (proprioceptive training) for patients with rheumatoid arthritis. Cochrane Database Syst Rev 2010:CD007648

8 American College Sports Medicine. ASCM's guidelines for exercise testing and prescription. 8th edn. Philadelphia: Wolters Kluwer, 2009.

9 Phillips EM, Kennedy MA. The exercise prescription: a tool to improve physical activity. $P M \& R$ 2012;4:818-25.

10 U.S Department of Health and Human Services. 2008 physical activity guidelines for americans secondary 2008 physical activity guidelines for Americans 2008. http://www.health.gov/paguidelines/guidelines/default.aspx (accessed $30 \mathrm{Dec}$ 2011).

11 Tiedemann A, Sherrington C, Close JC, et al. Exercise and sports science australia position statement on exercise and falls prevention in older people. J Sci Med Sport 2011;14:489-95

12 Shumway-Cook A, Woollacott M. Aging and postural control. In: Shumway-Cook A, Woollacott M, eds. Motor control: translating research into clinical practice. Philadelphia: Lippincott, Williams and Wilkins, 2007:212-32.

13 Berg KO, Wood-Dauphinee SL, Williams Jl, et al. Measuring balance in the elderly: validation of an instrument. Can J Public Health 1992;83(Supp 1):S7-11.

14 Duncan PW, Weiner DK, Chandler J, et al. Functional reach: a new clinical measure of balance. J Gerontol 1990;45:M192-M197.

15 Haines T, Kuys SS, Morrison G, et al. Development and validation of the balance outcome measure for elder rehabilitation. Arch Phys Med Rehabil 2007;88:1614-21.

16 Higgins J, Green S. Cochrane handbook for systematic reviews of interventions. 2011 www.cochrane-handbook.org 


\section{Systematic review}

17 The Centre of Evidence-Based Physiotherapy. PEDro scale. www.pedro.org.au/english/ downloads/pedro-scale/ (accessed 21 Jun 2011).

18 Sterne JA, Sutton AJ, loannidis JP, et al. Recommendations for examining and interpreting funnel plot asymmetry in meta-analyses of randomised controlled trials. BMJ 2011;343:d4002.

19 Higgins JP, Thompson SG. Controlling the risk of spurious findings from metaregression. Stat Med 2004;23:1663-82.

20 Gagnier JJ, Moher D, Boon H, et al. An empirical study using permutation-based resampling in meta-regression. Syst Rev 2012;1:1-9.
21 Review Manager (RevMan) [program]. 5.3 version. Copenhagen: The Nordic Cochrane Centre, The Cochrane Collaboration. 2014.

22 Harbord R, Higgins J. Meta-regression in Stata. The Stata J 2008;8:493-519.

23 Haas R, Maloney S, Pausenberger E, et al. Clinical decision making in exercise prescription for fall prevention. Phys Ther 2012;92:666-79.

24 Slade SC, Keating JL. Exercise prescription: a case for standardised reporting. $\mathrm{Br}$ 」 Sports Med 2012;46:1110-3.

25 Hoffmann TC, Glasziou PP, Boutron I, et al. Better reporting of interventions: template for intervention description and replication (TIDieR) checklist and guide. BMJ 2014;348:g1687. 